H I G H L I G H TS

CELL CYCLE

\section{Kinetochore fishing}

During mitosis, sister chromatids separate and are reeled into centrosomes at opposite poles of the spindle. The 'bait' that captures them is the kinetochore - a proteinaceous structure that binds both microtubules and centromeric DNA. Kinetochores move linked sister chromatids back and forth along the spindle during metaphase and separate sisters to the centrosomes during anaphase. One fundamental question is whether motor or non-motor microtubuleassociated proteins (MAPs) are the main factors responsible for microtubule attachment and chromosome movement. A group from Peter Sorger's lab now provides some answers - reporting in Cell, they present the results from a screen for kinetochore-associated factors.

To identify the microtubule-associated components of kinetochores, Sorger and colleagues exploited a phenomenon that occurs at metaphase - transient sister-chromatid separation - during which kinetochore proteins show a characteristic bilobed localization. They fused a collection of uncharacterized spindle proteins to green fluorescent protein (GFP) and then screened for this distinct localization pattern. From this, they found 11 candidate kinetochore components - ten structural factors and one kinase (Ipl1) that seems to regulate kinetochore function. To confirm that the localization of these factors reflects bona fide association with the kinetochore, they showed that their localization depends on an intact kinetochore.

The authors next asked whether these factors bind to centromeric DNA, as would be predicted for a structural component of the kinetochore. They expressed the GFP-tagged proteins in vivo, and then did chromatin immunoprecipitations. This confirmed that these factors bind centromeric DNA. The kinase Ipl1 did not bind in this assay, consistent with a role as a regulator rather than a structural component.

Intriguingly, the authors find that, whereas some factors (Ndc80, Spc24,
Spc25,

Dam1 and

Nuf2) localize predominantly to the kinetochore, others also localize to structures such as the spindle (Spc19, Spc34, Cin8 and Ipl1) or microtubulebased structures in the cytoplasm (Bik1 and Stu2).

So how does loss of these factors affect sister-chromatid separation? The authors find that a subset (Ndc80, Nuf2, Dam1, Stu2 and Ipl1) are required for the normal frequency of sister-chromatid separation. To characterize the type of defects that these mutants cause, the authors tagged chromatids with GFP and imaged live cells. Three types of defect emerged: chromosomes detach from the spindle and move randomly in the nucleus; chromosomes attach to a single pole but then cannot congress; or last, chromosomes attach at both poles but then move at reduced rates during metaphase. Given the different classes of phenotypes seen, the authors reasoned that several molecular machines with different mechanisms might drive chromosome movement and that there might be an ordered assembly of kinetochore components. They tested this and found that indeed, there is a hierarchy of dependencies for kinetochore association.

The conclusion, say the authors, is that the kinetochore is a multilayered structure, and that both motors and MAPs are required to form a fully functional kinetochore that can mediate microtubule-DNA interactions. Interestingly, they also note that the mitotic checkpoint — which checks that all kinetochores are correctly attached at both poles before anaphase can ensue - cannot be initiated without the presence of a kinetochore that can at least partly bind microtubules, supporting the idea that kinetochoreassociated factors themselves are the source of checkpoint-engaging factors. Alison Schuldt,

Associate Editor, Nature Cell Biology

Q) References and links ORIGINAL RESEARCH PAPER He, X. et al. Molecular analysis of kinetochore-microtubule attach-
ment in budding yeast. Cell 106, 195-206 (2001)
IN BRIEF

CELL SIGNALLING

Spred is a Sprouty-related suppressor of Ras signalling.

Wakioka, T. et al. Nature 412, 647-651 (2001)

Wakioka and colleagues describe the cloning and characterization of two receptor tyrosine kinase substrates, Spred-1 and 2, which contain an amino-terminal Ena/vasodilator-stimulated phosphoprotein homology-1 domain and a carboxy-terminal Sprouty-related domain. Like Sprouty — which inhibits epidermal- and fibroblast-growthfactor-mediated signalling - Spred suppresses the mitogenactivated protein kinase (MAPK) pathway. It does this by associating with Ras, thereby somehow suppressing the phosphorylation and activation of Raf.

\section{DEVELOPMENT}

Barentsz is essential for the posterior localization of oskar mRNA and co-localizes with it to the posterior pole.

Van Eeden, F. J. M. et al. J. Cell Biol. 154, 511-523 (2001)

During embryonic development, the localized translational activation of oskar messenger RNA at the posterior pole of the Drosophila oocyte defines where the abdomen and germ cells form. This localization is thought to involve active transport along the cytoskeleton, but how the mRNA is coupled to this process remains unknown. Van Eeden and colleagues report the phenotypic and molecular characterization of a novel locus called barentsz, which co-localizes with oskar mRNA and is specifically required for the movement of oskar mRNA from the anterior to the posterior of the oocyte. This suggests that Barentsz is essential for the posterior localization of oskar mRNA and behaves as a specific component of the oskar RNA transportation process.

\section{TRANSLATION}

\section{The path of messenger RNA through the ribosome}

Yusupova, G. Z., Yusupov, M. M., Cate J. H. D. \& Noller, H. F. Cell 106, 233-241 (2001)

The ribosome translates messenger RNA (mRNA) into proteins but the molecular mechanisms that influence the unwinding of the mRNA secondary structure and correct reading of the message have remained unknown. Yusupova and colleagues have identified these processes by mapping the complete path of the mRNA through the 70S ribosome of Thermus thermophilus using X-ray crystallography. They report that the mRNA is threaded through a channel that wraps around the neck of the 30 S subunit and that the locations of downstream regions of the mRNA in the ribosome have implications for translational initiation, frame shifting and other functional interactions. The authors predict that mRNAs will follow similar paths in eukaryotic ribosomes and hope their findings will provide a basis for designing strategies to test emerging models for the mglecular basis of ribosomal dynamics. 